\title{
The Usefulness of HIV-1p24 in Detecting the Presence of HIV Infection in Histopathology Tissue Specimens
}

\author{
Felix Emeka Menkiti ${ }^{1} \quad$ Cornelius Ozobia Ukah \\ 1Department of Anatomic Pathology and Forensic Medicine, \\ Nnamdi Azikiwe University, Nnewi Campus, Anambra State, \\ Nigeria \\ 2Department of Morbid Anatomy and Forensic Medicine, \\ Obafemi Awolowo University Teaching Hospitals Complex, \\ Ile-Ife, Osun State, Nigeria \\ Asian J Oncol:2021;7:40-44
}

Kayode A. Adelusola ${ }^{2}$ Ifeoma Florence Ezejiofor ${ }^{1}$

\begin{abstract}
Address for correspondence Felix Emeka Menkiti, MBBS, FMCPath, Department of Anatomic Pathology and Forensic Medicine, Nnamdi Azikiwe University, Nnewi Campus, PMB 5025, Awka, Anambra State, Nigeria (e-mail: fe.menkiti@unizik.edu.ng).
\end{abstract}

\begin{abstract}
Keywords

- detection

- histopathology tissues

- HIV-1p24

- human

immunodeficiency virus

- immunohistochemistry
\end{abstract}

Introduction HIV (human immunodeficiency virus)-associated immunodeficiency is the single most important acquired immunodeficiency in humans, underlying several diseases. The presence of HIV infection may be unsuspected or undetected in tissues due to latency or failure of the consulting physician to document it on the requisition form. HIV-1p24 protein is a component of the HIV particle capsid that can be exploited serologically and immunohistochemically to detect the presence of HIV infection. This study aimed to assess the usefulness of HIV-1 p24 immunohistochemistry in detecting the presence of HIV infection in histopathology tissues in a resource-challenged setting.

Materials and Methods HIV-1p24 immunohistochemical expression was evaluated in 59 cases from the archives of both Nnamdi Azikiwe University Teaching Hospital (NAUTH) and Pathocon Specialist Clinic and Research Institute over a 10-year period. Pathology consult forms of all clinically and morphologically suspected cases of Kaposi sarcoma and their blocks were retrieved, recut, and reviewed using hematoxylin and eosin, and then subjected to HIV-1p24 immunohistochemical staining.

Results Of the 59 cases, 38 expressed p24, whereas 21 were negative. Fifteen of the positive cases had no prior documented evidence of HIV infection. Five cases with clinically known statuses, four positive and one negative, were found to be negative and positive, respectively, following HIV-1p24 immunohistochemical staining, probably due to usage of ELISA (enzyme-linked immunosorbent assay) detection kit only and long term antiretroviral therapy. HIV infection was commoner in the third and fourth decades of life.

Conclusion HIV-1p24 is a useful low-cost immunohistochemical marker in detecting the presence of HIV infection in histopathology specimens, even in previously unsuspected cases. published online September 17, 2020
DOI https://doi.org/

$10.1055 / \mathrm{s}-0040-1718979$

ISSN 2454-6798. (c) 2020. Spring Hope Cancer Foundation \& Young Oncologist Group of Asia.

This is an open access article published by Thieme under the terms of the Creative Commons Attribution-NonDerivative-NonCommercial-License, permitting copying and reproduction so long as the original work is given appropriate credit. Contents may not be used for commercial purposes, or adapted, remixed, transformed or built upon. (https://creativecommons.org/licenses/by-nc-nd/4.0/) Thieme Medical and Scientific Publishers Pvt. Ltd., A-12, 2nd Floor, Sector 2, Noida-201301 UP, India 


\section{Introduction}

The human immunodeficiency virus 1 (HIV-1), the main causative agent of acquired immune deficiency syndrome (AIDS), was discovered in $1983 .{ }^{1}$ Since its discovery, it has become a global pandemic, having infected more than 70 million people and claiming more than 35 million lives. ${ }^{2,3}$ HIV-1 is polymorphic in nature, with many genetically related variants, due to the complexity of its cDNA formation and its mutation-prone reverse transcriptase enzyme.,5 The changes in its biological characteristics, including cytopathic ability, immune evasion, coreceptor usage, and tropism, are related to the evolution of the viral genome and the host factors. ${ }^{4}$ As the single most important immunodeficiency in human, HIV-associated immunodeficiency manifests as AIDS in its most severe cases. ${ }^{6}$ AIDS is associated with several opportunistic infections and malignancies. ${ }^{3,7,8}$

Despite the introduction of the highly active antiretroviral therapy with the reduction in viremia to often undetectable levels, complete eradication is still a mirage. ${ }^{8}$ This could be accounted for by the generation of viral reservoirs, including some resting CD4+ memory and naive $\mathrm{T}$ cells and macrophages that sequester the virus within tissues of infected persons. ${ }^{9-11}$ These latently infected cells can escape detection and destruction by host defenses, and therefore infection spread in and between individuals. ${ }^{12}$

Several procedures and technologies including polymerase chain reaction have been developed to detect and quantify the virally infected reservoir cells, which cannot be detected through serology, but these were suboptimal in doing so. ${ }^{9}$ This may be attributed to interpretation and technical difficulties of the detection systems, including the need for large amounts of blood, extensive time allocation, high cost, and significant differences in assay sensitivity. ${ }^{13}$

Pathologists' role in the management of HIV-related disease is crucial. However, his role requires provision of properly filled pathology requisition form by the clinicians: this is a great challenge in our setting. ${ }^{4}$ Also, the early stages of HIV infection may be accompanied by little or no clinical abnormalities such that the pathologist encounters tissue specimens from HIV-infected patients with no previous clinical suspicion or information of HIV infection. ${ }^{15}$ In a resource-poor setting like ours, accessing the high-tech procedures to confirm HIV as an etiologic agent is difficult. Moonim et al reported that HIV-1 p24 immunohistochemical staining of histopathology tissue samples is a useful method for establishing HIV infection in clinically suspected cases and when clinical suspicion is absent or low. ${ }^{15}$ This study, therefore, aims to assess the usefulness of HIV-1 p24 immunohistochemistry, a relatively low-cost procedure, in detecting the presence of HIV infection in tissue specimens using formalin-fixed paraffin-embedded (FFPE) tissue blocks in a resource-challenged setting.

\section{Materials and Methods}

We retrieved all the request forms and FFPE tissue blocks of cases that were clinically and morphologically suspected to be Kaposi sarcoma ([KS] an AIDS-defining malignancy) using hematoxylin and eosin (H\&E) over a 10 -year period (January 2007 to December 2016) in the two specialist histopathology laboratories located in Nnewi, Anambra State, Nigeria: the Histopathology Department of Nnamdi Azikiwe University Teaching Hospital (a federal teaching hospital) and Pathocon Specialist Clinic and Research Institute (a well-staffed private pathology laboratory/ diagnostic center established in 2007 by two pathologists with a good documentation system). Cases with poorly preserved or missing tissue blocks as well as tissue blocks with inadequate tissue left for sectioning were excluded from the study.

Fresh sections were made from the tissue blocks and stained with H\&E stains and reviewed using binocular compound light microscope (Carl Zeiss).

Immunohistochemical studies were conducted by the indirect immunoperoxidase method on the formalin-fixed paraffin-embedded (FFPE) tissue blocks. Four-micrometerthick tissue sections were made from the selected FFPE tissue blocks, deparaffinized by passing it through xylene, and then rehydrated in decreasing alcohol concentrations and mounted on positively charged glass slides. Antigens were retrieved from the tissues by heat-induced epitope retrieval method using a pressure cooker. Endogenous peroxidase activity was blocked using $3 \%$ hydrogen peroxide. The tissues were then incubated with the primary antibody, rinsed, and then followed by the use of secondary detection system using diaminobenzene as chromogen. Immunohistochemical staining was performed using HIV-1 p24 (HIV particle capsid, Dako, dilution 1:10, 30 minutes of incubation) with control in parallel. All steps were performed at room temperature.

\section{Interpretation of Results}

HIV-1 p24 was expressed in the cytoplasm of the positive cells and was interpreted as "positive" when there is distinct "cytoplasmic or pericytoplasmic" staining of $>10 \%$ of the infected cells, but it was considered as "negative" when the cells exhibit expression of HIV-1 p24 "cytoplasmic or pericytoplasmic" staining in $<10 \%$ of these cells. A known HIV positive tissue that stained positive for HIV-1 p24 was used as control.

\section{Data Analysis}

The data analysis was conducted using the Statistical Package for Social Sciences (SPSS) software Version 20.0 (IBM Corp.), and the result presented are with tables and charts.

\section{Results}

The 59 cases that met the inclusion criteria were reviewed and subsequently subjected to HIV-1 p24 antibody staining protocol to detect the presence of HIV infection.

Of the 59 cases, 38 (64.4\%) had information on the clinical HIV status of the patient written on the request forms, whereas $21(35.6 \%)$ were not specified (-Table 1). All were subjected to HIV-1p24 immunohistochemistry and identified as cytoplasmic or pericytoplasmic staining of the infected cells, especially the dendritic cells and other antigen-presenting cells of the different tissues ( $\mathbf{- F i g}$. 1A-C). Of the 59 cases, 
Table 1 Clinical HIV status and HIV-1 p24 immunohistochemistry

\begin{tabular}{|l|l|l|l|l|}
\hline \multicolumn{2}{|c|}{} & \multicolumn{2}{|c|}{ HIV-1 p24 immotal } \\
\cline { 2 - 5 } \multicolumn{2}{|c|}{} & Positive & Negative & \\
\hline \multirow{3}{*}{ HIV status } & Positive & 22 & 4 & 26 \\
\cline { 2 - 5 } & Negative & 1 & 11 & 12 \\
\cline { 2 - 5 } & Not specified & 15 & 6 & 21 \\
\hline Total & & 38 & 21 & 59 \\
\hline
\end{tabular}

Abbreviation: HIV, human immunodeficiency virus.
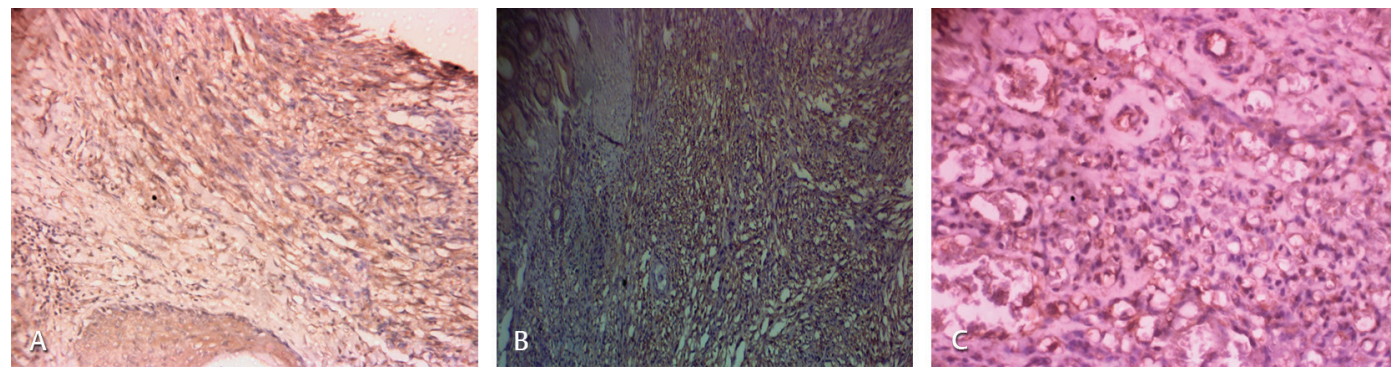

Fig. 1 Human immunodeficiency virus 1 (HIV-1) p24 immunohistochemical staining photomicrographs of some of cases that were positive to HIV-1 p24 immunohistochemical staining. (A) Cutaneous Kaposi sarcoma (KS) (100×). (B) Intestinal KS (100×). (C) HIV-1p24 staining of bacillary angiomatosis (100x).

Table 2 Clinically unspecified cases with HIV-1 p24 positivity

\begin{tabular}{|l|l|l|l|l|}
\hline S. no. & Age/sex & Site & Clinical diagnosis & Morphological diagnosis \\
\hline 1 & $60.0 / \mathrm{M}$ & Lower limb & Not specified & Kaposi sarcoma \\
\hline 2 & $27.0 / \mathrm{F}$ & Upper limb & Wart & Kaposi sarcoma \\
\hline 3 & $33.0 / \mathrm{F}$ & Head and neck & $\begin{array}{l}\text { Dermatosis to exclude granuloma } \\
\text { pyogenicum }\end{array}$ & Kaposi sarcoma \\
\hline 4 & $51.0 / \mathrm{M}$ & Lower limb & Not specified & Kaposi sarcoma \\
\hline 5 & $48.0 / \mathrm{M}$ & Lower limb & Kaposi sarcoma & Kaposi sarcoma \\
\hline 6 & $22.0 / \mathrm{M}$ & Lower limb & Mole & Bacillary angiomatosis \\
\hline 7 & $26.0 / \mathrm{M}$ & Genitals & Kaposi sarcoma & Molluscum contagiosum \\
\hline 8 & $53.0 / \mathrm{F}$ & Intestinal & GlST & Kaposi sarcoma \\
\hline 9 & $51.0 / \mathrm{F}$ & Tongue & Kaposi sarcoma & Bacillary angiomatosis \\
\hline 10 & $46.0 / \mathrm{F}$ & Trunk & Kaposi sarcoma & Lupus vulgaris \\
\hline 11 & $46.0 / \mathrm{M}$ & Generalized & Not specified & Kaposi sarcoma \\
\hline 12 & $70.0 / \mathrm{F}$ & Lower Limb & Wart & Kaposi sarcoma \\
\hline 13 & $65.0 / \mathrm{M}$ & Lower limb & Hemangioma & Kaposi sarcoma \\
\hline 14 & $43.0 / \mathrm{F}$ & Lower lip & Epulis & Kaposi sarcoma \\
\hline 15 & $31.0 / \mathrm{F}$ & Trunk & Nevus & Kaposi sarcoma \\
\hline
\end{tabular}

Abbreviations: F, female; GIST, gastrointestinal stromal tumor; HIV, human immunodeficiency virus; M, male.

38 were positive for the HIV1 p-24 immunohistochemistry (-Table 1).

Of the 38 positive cases, 22 (57.9\%) patients including 11 females and 11 males were known to have had HIV infection at the time of their biopsy, whereas HIV-1p24 immunoreactivity was the first indication of HIV infection in 15 of the positive cases. These 15 unspecified but HIV-1p24 positive cases are listed in - Table 2. However, 1 out of the 12 negative cases was positive to HIV-1p24 immunohistochemical staining (- Table 1). The modal age group and median age for HIV infection were third decade of life and 37.5 years, respectively ( - Fig. 2 ).

\section{Discussion}

Immunodeficiency disorders are characterized by aberrant immune regulation and marked by an increased risk for the development of several disease entities 


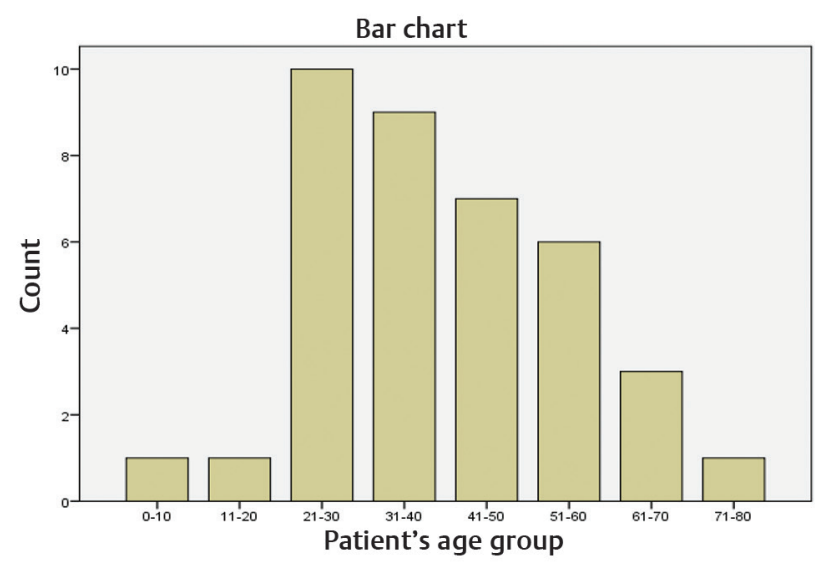

Fig. 2 Bar chart illustrating HIV distribution by age. HIV, human immunodeficiency virus.

including malignancies, infections, and autoimmune diseases. ${ }^{16}$ The HIV-associated immunodeficiency is the single most important acquired immunodeficiency in human ${ }^{6}$ and is associated with several opportunistic infections and malignancies. ${ }^{7.8}$ Few studies have shown that HIV infection can be detected in histopathological tissue specimens even in clinically unsuspected cases..$^{15,17}$ This index study is the first of its kind in Nigeria to detect HIV infection in histopathology tissue specimens using HIV-1p24 immunohistochemical stain after extensive search in PubMed and Google Scholar.

HIV-1p24 protein is a component of the HIV particle capsid, which can be detected in patient's blood as early as 2 weeks after HIV infection, even prior to the development of anti-HIV antibodies, and it is therefore necessary to accurately detect the HIV status of the patient. ${ }^{15}$ Immunohistochemically, HIV-1 p24 has been shown to be useful in confirming the presence of HIV in tissue specimens from lymphoid and nonlymphoid organs and can detect unsuspected HIV infection in these organs on histopathological material..$^{15}$ Therefore, we sought to investigate the utility of HIV-1 p24 in detecting HIV infection in FFPE tissue blocks.

In this study, 38 of the 59 cases assessed were positive to the HIV-1p24 immunohistochemical stain. Of these, 15 were clinically unspecified cases because the HIV statuses were not indicated on the request forms. Hence, the first indication of HIV infection to the pathologists on these cases was HIV-1p24 immunoreactivity after H\&E-stained diagnoses of the lesions. Hence, HIV-1 p24 immunostaining is an effective tool in the identification of HIV as an etiologic agent in surgical specimens.

Moonim et al demonstrated the usefulness of HIV-1p24 immunohistochemistry in identifying HIV infection even in clinically unsuspected cases when they examined 123 tissues from both lymphoid and nonlymphoid organs. ${ }^{15}$ This index study has also proved that HIV-1p24 immunohistochemistry is useful in our setting where access to costly conventional procedures is limited. This study also emphasizes the usefulness and suitability of archival tissue blocks in HIV-1p24 immunohistochemistry.
In addition, we discovered in this study that five cases with stated clinical HIV status (four positive and one negative) stained paradoxically with HIV-1 p24 immunostain. A review of the information on the histopathology consult form showed that these were outpatient referrals. The possibility that the HIV status in these cases was assessed using ELISA (enzyme-linked immunosorbent assay) or other HIV antibody detection test, but not Gag p24 serology, may explain this discordance. It is also possible that the biopsy was taken from a patient who has been on long-term antiretroviral therapy.

It is documented in the literature that HIV-1p24 expression may not be detected in a subset of patients on long-term antiretroviral therapy. ${ }^{18}$ Moonim et al in their study noted five cases (mostly referral cases with limited information) with prior serological evidence of HIV infection that stained negative to HIV-1p24 immunohistochemistry: one was a tissue from a patient with well-controlled disease (high CD4 counts and undetectable virus in the blood for the past 5 years); hence, the possible loss of p24 expression due to undetectable viral load could be an explanation for this. ${ }^{15}$ However, they could not provide an explanation for the rest discordant results.

HIV infection in our index study occurred commonly in the third and fourth decades of life. This corresponds with the data from Nigerian National Progress report on AIDS control, indicating the third and fourth decades of life as the peak age for HIV infection diagnosis in Nigeria. ${ }^{19}$

\section{Conclusion}

HIV-1p24 immunohistochemical staining is a useful cost-effective tool for detecting the presence of HIV infection in FFPE tissue blocks, even in clinically unsuspected and undetected cases.

\section{Conflict of Interest}

None declared.

\section{References}

1 Gryseels S, Watts TD, Kabongo JM, et al. A near-full-length HIV-1 genome from 1966 recovered from formalin-fixed paraffin-embedded tissue. bioRxiv 2019 (e-pub ahead of print). doi: $10.1101 / 687863$

2 World Health Organization. WHO Global Health Workforce Statistics. Available at: http://www.who.int/hrh/statistics/ hwfstats/en. Accessed January 5, 2020

3 Zhang Q, Frange P, Blanche S, Casanova JL. Pathogenesis of infections in HIV-infected individuals: insights from primary immunodeficiencies. Curr Opin Immunol 2017;48:122-133

4 Naif HM. Pathogenesis of HIV Infection. Infect Dis Rep 2013; 5(Suppl 1):e6

5 LamersSL,SalemiM,GalliganDC,etal.ExtensiveHIV-1 intra-host recombination is common in tissues with abnormal histopathology. PLoS One 2009;4(3):e5065

6 Morens DM, Folkers GK, Fauci AS. The challenge of emerging and re-emerging infectious diseases. Nature 2004;430(6996): 242-249

7 Carbone A, Vaccher E, Gloghini A, et al. Diagnosis and management of lymphomas and other cancers in HIV-infected patients. Nat Rev Clin Oncol 2014;11(4):223-238 
8 Yarchoan R, Uldrick TS. HIV-associated cancers and related diseases. N Engl J Med 2018;378(11):1029-1041

9 Eugenin EA, Berman JW. Improved methods to detect low levels of HIV using antibody-based technologies. Methods Mol Biol 2016;1354:265-279

$10 \mathrm{Xu} \mathrm{W}$, Li H, Wang $\mathrm{Q}$, et al. Advancements in developing strategies for sterilizing and functional HIV cures. BioMed Res Int 2017;2017:6096134

11 Coleman CM, Wu L. HIV interactions with monocytes and dendritic cells: viral latency and reservoirs. Retrovirology 2009;6:51

12 Embretson J, Zupancic M, Beneke J, et al. Analysis of human immunodeficiency virus-infected tissues by amplification and in situ hybridization reveals latent and permissive infections at single-cell resolution. Proc Natl Acad Sci U S A 1993;90(1):357-361

13 Spina CA, Anderson J, Archin NM, et al. An in-depth comparison of latent HIV-1 reactivation in multiple cell model systems and resting CD4+ T cells from aviremic patients. PLoS Pathog 2013;9(12):e1003834

14 Soyemi SS, Faduyile FA, Sanni DA, et al. Completeness and adequacy of clinical and demographic information in non-gynaecologic surgical pathology requisition forms: an analysis of 1046 cases. Niger J Med 2019;28(1):1-4
15 Moonim MT, Alarcon L, Freeman J. Mahadeva U, van der Walt JD, Lucas SB. Identifying HIV infection in diagnostic histopathology tissue samples-the role of HIV-1 p24 immunohistochemistry in identifying clinically unsuspected HIV infection: a 3-year analysis. Histopathology 2010;56(4):530-541

16 Heise ER. Diseases associated with immunosuppression. Environ Health Perspect 1982;43:9-19

17 de Paiva GR, Laurent C, Godel A, et al. Discovery of human immunodeficiency virus infection by immunohistochemistry on lymph node biopsies from patients with unexplained follicular hyperplasia. Am J Surg Pathol 2007;31(10):1534-1538

18 Alòs L, Navarrete P, Morente V, et al. Immunoarchitecture of lymphoid tissue in HIV-infection during antiretroviral therapy correlates with viral persistence. Mod Pathol 2005; 18(1):127-136

19 National Agency for the Control of AIDS. United Nations General Assembly Special Session (UNGASS) Country Progress Report. Available at: https://data.unaids.org/pub/report/2010/ nigeria_2010_country_progress_report_en.pdf. Accessed June 10,2020 